\title{
The Shape of the World: \\ What if Aesthetic Properties Were Real?
}

\author{
Crispin Sartwell
}

\begin{abstract}
Aвstract Perhaps we should entertain the idea that aesthetic properties are no less (but no more) objective than properties like weight or shape. Indeed, the weight and shape of something are themselves aesthetic properties of that thing. And we might speculate or (what the heck) assert that aesthetic properties are no more (but no less) socially constructed than size or material composition, for example. Indeed the size and material composition of something are aesthetic properties of it. We might, that is, live in an aesthetic universe, live embedded in an aesthetic reality. Then, for example, to give a full description of any thing or phenomenon, we would have to resort to aesthetic categories: perhaps there is no natural science, for example, without aesthetics, and vice versa. On a good day, the universe might really, actually, truly be beautiful. KEYWORDS Aesthetic properties, universe, materialism, materiality of art
\end{abstract}

So here is how to think about the universe. It is sort of a fabric; or, better, a skein, or perhaps a tangle, snag, snarl, mesh, mess of rough twine, tied back on itself in many knots of many kinds. It is a network or grid of knots, we might say, but the arrangement is not fully comprehensibly ordered. As interpreted from within our cognitive limitations, it is chaotic rather than Cartesian.

I am going to use "skein" as a quasi-technical term expressing the midpoint between a sheer mess or pile of tangled, knotted string and a Cartesian grid. The world consists of many strings or ultimately a single string tied together or back on itself, forming something that looks like a fabric at a distance, consisting of myriads of knots closer up. Each knot is "an individual" - a person, tree, refrigerator, county. Each knot has a distinct form and location and physical composition (that is, the [token] portion of material in which it consists is different from that of any other). But it is itself absolutely nothing but a set of relations to other portions of the skein: it consists without remainder of string in connection. Different points of view on the skein produce different impressions, so that at a wider angle larger structures emerge: clusters of knots, etc. But nevertheless the skein itself does not depend on any point of view, cultural practice, interpretation, description, though groups of people operating in a practice are themselves a knot of knots in connection or conversation.

Emerson says: "A man is a bundle of relations, a knot of roots, whose flower and fruitage is the world. His faculties refer to natures out of 
him."1 It is true that "the fabric of reality" is an old saw or the merest cliché; you keep running into it in everything from Confucius to the Greeks to the latest popularization of string theory or what I am calling knot theory: the theory of entanglements: universe as macramé or crochet. From one point of view, the fact that I am harping on a cliché is a drawback. From another, it suggests that I have latched on to some fundamental insight.

With regard to the science or everyday experience of the knotscape, we might say that you could pick out indefinitely many structures or introduce various ontological ordering principles for various purposes: taxonomies of knots, identifications of sub-knot elements to show the structure of particular knots (precisely the function of skein analysis in knot theory). But for a given claim to be true - for us to say something true about the universe - entails that the skein actually has the structure attributed to it. In some sense, what counts as a knot has to be fixed and is a question: "is that tangle a single knot or a stack of knots or not a knot at all?", which is always dependent on a particular standpoint that is being taken up, or the rough ontology.

The way we order the array into individuals, however, is not whimsical or merely conventional. Indeed, if we did not order our experience of the world more or less the way the world actually is, we would long ago have been extinguished. We order the world from within the world, as part of the world. If we did not distinguish lions from various portions of its environment, we would merely be prey for something in turn able to distinguish us. Nevertheless, of course, the body of the lion or the human being is not fully distinct from its surround, and as the lion is breathing, seeing, smelling, eating, excreting, growing, aging, it is in interchange. We are actual creatures functioning in an actual environment; we are of necessity continuously responsive to actual features of it in their emergence through us. We have to "mirror" reality, we might say, but I see attention and perception as much more intimate than that: as an actual taking-in of external objects: perception is ingestion.

Like many people, I at once use or even glorify the term "nature" and suspect it of harboring the entire environmental problem, wrapped up like a seed. But if "nature" means anything, then it means the whole of the skein, whatever in turn that might mean: the skein as it really is under all dimensions of experience and analysis, in all its relations. So what are these dimensions? If I were trying to issue a description of the skein where would I begin? If I were trying to issue an ideally replete or 
full description, what would I have to include? And I am of course again speaking from within the world - I am included in what I am describing - and my speaking in it of it itself is a deformation of or a tug on it, a material event.

If I said, for example, that this skein or tangle displayed aesthetic properties such as (for example) beauty, what would I be saying? Or I might point or mark out certain passages, knotscapes as it were. Maybe I like it when they fall into a regular or predictable repeating pattern; or maybe I like fantastic concatenations of string. Maybe I reach up and make adjustments, in illustration of some point, or just as a matter of more or less arbitrary preference. So if I was trying to give a description of the skein, I might resort to aesthetic qualities as holistic sensible qualities of particulars, regions, or the skein as a whole. As significantly, aesthetic values may be guides for interventions in the knotscape: ways of tying, etc.

If I said of any such thing or array that it was "beautiful," you could take this to denote approval, but typically there is also more that I could say, and there is certainly more that I could say if I am a professional: about in virtue of what qualities it is beautiful: about what makes the pebble or the painting or interstellar space beautiful. Well, essentially we are in this position with regard to all qualities; they are qualities, as it were, of the ontological object that consists of a culturally embedded perceiver/speaker/body in juxtaposition with the thing being experienced or described, suspended in juxtaposition in an atmosphere or tangle of air and light and language.

There are - let us say: provisionally or apparently - three salient moments or zones or aspects of the act of asserting of something that it is beautiful. There is the object as it exists outside the interpreter (let us suppose) in space (let us suppose). There is the perceiver of whatever sort. And there is a set of vocabularies and, more widely, social practices that have to do with beauty. Another language might have another word with a different inflection, and of course other peoples or people might have different "standards" of taste in whatever dimension, perhaps an elaborate system within a system of elaborate systems different from one's own. Now I start by distinguishing these three dimensions or aspects - the objective, the subjective, and the social - because that reflects our way of scientifically, social-scientifically, philosophically accounting for these things. But the idea that "physical reality," "social practices," and "subjectivities" can actually be distinguished in a principled way is the very notion I want to destroy.

Twentieth century philosophy took it upon itself to collapse the dis- 
tinction between the individual and the social: you see this in the work, for example, of Mead, Wittgenstein, or Foucault. And you saw it in Rousseau, Hegel, German nationalism, and Marx, for example. Now, I think that this collapse took a distorted form; it just reduced the individual to the social, whereas the collapse should have been mutual and simultaneous. But at any rate, having collapsed the social/individual distinction, we should proceed to destroy the distinctions between the material surround - that is, nature, the objective - and the social, and between nature and the individual. Bruno Latour's work should be exemplary here, and his view that each object which sociologists could study is a "lash-up" of (what have been considered) social, individual, and material factors, finally motivates a desire to completely forego/destroy all the concepts involved, particularly "nature" and "the social." "The social" is a version of what is also called "the artificial," the fabricated or man-made, and of course "the artificial" is the proper complement to nature, so that finally the term "nature" only registers our own apartness from the world; nature is itself an artifact of this artifice, "nature." This is why I say the whole environmental problem is wrapped up in the concept. Whether nature is the garden from which we have fallen, or the merely material over which we as spirits have dominion, the human and the rest of the material world are bifurcated in a false but potentially conceptually fatal way. In particular, the social is opposed to the merely or the rawly material; "social" explanations of race, gender, and so on, resist precisely the naturalization of various socially-determined vocabularies or taxonomies.

At any rate, if I use the term "nature," and I may, I intend to pick out absolutely everything, including everything we are as human beings and everything we have made and every intervention we have performed in reality. Really, we have no idea what the universe would be without us in it or with only us in it. Of course, this idea of talking about the whole or the one without the other may be nonsense, and is itself apparently outside the set of which it is a member. But on the other hand I do not think we are going to be able to stop talking about the whole: we are so adept at the encompassing abstraction that the ultimate encompassing abstraction beckons irresistibly. And among other things, when we talk about the whole, or when we order into wholes including individuals, we are entering aesthetic territory.

Latour argues that we have to replace the social in the surround of the physical: there are no people, practices, institutions, conventions, truths without non-human things. The social is entirely embedded 
in "the material," "nature," the non- or less-than or more-than human. Without physical objects, he points out, "the social" is an entirely inert mysterious powerless essence that does not do or explain anything. I associate myself with these remarks.

Now, when we think of the aesthetic properties of an object, we may think of its form, or qualities of its form. If form were to be understood, or could be thought, apart from matter, then this would indicate that in some sense the aesthetic properties of a thing are not, as it were, real properties of it; not, for example, "objective" or scientifically ascertainable or studyable properties. But on the contrary, the form of something is precisely its material arrangement (here I think we are close in some ways to the dispute between Plato and Aristotle on "form."). The Parthenon, we might say, is that very material under that very configuration. At each actual site, form and material are identical. Every actual object is a site at which matter and form coincide: they coincide at that site, and neither ever appears independently at any site, that is, in any object. Skein analysis describes possible knot configurations in an "abstract" way; but the description is a concrete set of inscriptions or image-tokens, and the knot is made of string.

If we believe that, at a minimum, to describe the form of something is to describe that very thing, which is a material object, then insofar as the aesthetic aspects are the formal aspects, the aesthetic aspects are germane and material. So for example, of the skein, the question whether it is as a whole well or comprehensibly ordered: is that a scientific question or an aesthetic question? Well, I think that is precisely the sort of thing you hope to find out, detect, or even adore in the practice of science. What would it mean for a system to be well-ordered? Various dimensions of fact-value suggest themselves; one is surely aesthetics. We might say that the epistemic standards of science are aesthetic through and through, that the practice demands a standard of rationality that we might call an aspiration to or a reflection of an experience of beauty, its trace.

For example, we might think of an ordering principle like Ockham's razor: a nice way to cut cognitive costs, or maybe not, but above all a standard of beauty in explanation. Indeed the standard is identical to Hutcheson's: the compound ratio of variety to uniformity: individuals comprehended under the single category in taxonomy. Ockham's razor gave us both materialism and idealism, opposed to the death, aufhebunged in Hegel and so on, but both committed to the ultimate singleness of the universe, the coherence of all in a single ontological plane. That is, though the materialists and idealists of the modern period disagreed about ontology, they agreed 
about aesthetics, and that is precisely why even Berkeley at times threw up his hands and said the dispute was verbal: as long as you give me these things - this world, ordered under this aesthetic - I do not really care whether you call them material or mental. He certainly believed that once one no longer believed in the material world, one would just keep doing science the same way as always: an extraordinary idea considering the usual association of science with materialism throughout the nineteenth century.

Consider a shadow as an object. It is best conceived as a situation, or an aspect, feature, portion of a situation. We might say that the shadow is caused by the light source, object, etc., but in truth it is a mercurial chunk of that situation: the shadow is not called into being by the light, etc.: it is the light in its flow, implicating an environment and certain sorts of sensory apparatus. The "modern" account of human consciousness as a "sensorium" or an arena of "ideas" conceived primarily as mental images - the basic notion underlying both "rationalism" and "empiricism" - reifies the shadow, isolates it, severs its connections, or actually deletes the situation that makes it possible and the material of which it consists.

We ought to think of human perception as a penetration of the body by the world: a strand going in and helping to compose the knot, and then emerging again and on to the next. When I see something, light literally enters my body and works its way through it in a series of transformations: my act of perception encompasses an external-world situation, or is itself an external/internal world situation. My body/my consciousness is composed of stuff appropriated from the environment; it is not distinct from the environment in any sense. My consciousness is a trace or shadow in, or better, a knot of, a physical reality.

The same is true of the social both ways round. So first of all, all these individual events/situations of perception are parts of the social. My consciousness is a portion of the social as a knot is part of a larger section of skein, or indefinitely many larger sections. But social vocabularies, narratives, descriptions, and so on, are massively constrained by a physical universe. That social systems in some sense emerge from physical environments is a commonplace, though no doubt controversial in the sense that a linguistic idealist such as Rorty would not even give me the term "physical environment" or would regard it as an artifact of social practices, language games, and the like. Well, it is an artifact of social practice, but no more than social practices are artifacts of it. Again Latour's work is exemplary here, and he shows minute 
by minute, detail by detail, how social practices are continuously embedded and re-embedded in physical objects, as they transform those objects according to their recalcitrances, are transformed by those objects according to our own recalcitrances, and so on.

Truth, we might say provisionally, is a snarl of such portions or aspects, their mutual compromise or annihilation into each other. So "the tree is beautiful" implicates the social in, for example, a romantic celebration of nature (behind it lurks Muir or Thoreau or Wordsworth etc: a whole history and vocabulary of appreciation characteristic of big swathes of culture). It implicates the social for that matter, in that "beautiful" is a word and there are no private languages. "Tree" is a word too, believe it or not. That it picks out what it does and fails to pick out what it does not is not any individual's decision. Perhaps it implicates the social in that, in emitting the utterance, I am trying to do something with you or to you: impress you, agree with you, attack you, distract you. Of course, "the tree is beautiful" notoriously implicates the individual in her subjectivity; in fact, some thinkers make it merely a trace or expression of a supposedly purely subjective experience, a variety of pleasure for example. This, as even its advocates such as Santayana have seen, appears to make sentences like "The tree is beautiful" sheer mistakes, since it is on this account not about a tree at all. But at any rate, entirely delete the "individual" or "subjective" dimension of the experience and it is senseless to talk about beauty. But I also insist on this - and here I call on the shades of Muir, Thoreau, and Wordsworth to testify - when I say "the tree is beautiful," I am talking about the tree, not about myself. "The tree is beautiful" does not mean "I feel funny."

As to the relation of the physical to the social, we deal with things and materials themselves with regard to culturally circulating meanings, while the physical features of things and materials massively shape those very meanings. For example, the stone slabs that form the floor and counter of an old-fashioned small-town bank convey stability and quiet wealth. But the stone is fitted in virtue of its physical features to express such things; its semiotics is not a mere stipulation; it was selected for the task in virtue of the fact that it was physically suited to convey such meanings. Stone is more stable, enduring, and heavy than bakelite or linoleum, for example. If we think of the stone in its physical qualities, however, we cannot detach those from the social entirely. The stone has to be inexpensive enough to be budgetable when bakelite is available. But then the cost of the stone depends on a myriad of social factors, including the activities of the central bankers, for example. 
The stone has to be workable, which depends on the tools and skills at our disposal. But the way that this particular kind of stone enters the market and gets worked - the social uses of stone - has a myriad of physical aspects: how common the stone is on earth, how accessible the quarries are to the bank's location, and then what transportation resources we have, what materials we have available to make the tools needed to work the stone, and so on and on. And someone had to make the decision to install, someone who had mastered the semiotics of stone and also had some handle on the material juxtapositions that would make it possible to install it. Someone designed the installation. You cannot explain anything - including the aesthetic qualities of the stone or bank - by the merely social. You cannot explain them by the merely material. You cannot explain them as merely personal. The meaning and the physical quality of the installed stone is an incredibly elaborate concatenation or lash-up of factors.

Meaning is neither only out there among things themselves nor only in the head or the language, but is an interaction of persons and environments, physical or virtual, stone or televised image. The attribution of an aesthetic feature to a thing involves a language and a culture and an experiencer, but if it is true, it picks out a real feature of that thing in its context. Attributions of aesthetic properties to things are not merely objective, not merely subjective, and not merely culturally fixed: the aesthetic features of a thing are features of it in a situation, which implicates all of these, in every case. We might allow this to show us that the distinctions between the personal, the social, and the material simply cannot be maintained. It is skein all the way across.

A building or a city section might resolve roughly into a triangle, be symmetrical or balanced; it might have been made of local stone, or concrete and steel, or whatever can be salvaged from the local dump. And it will have what we might think of as art-historical properties, which are also underlain by formal properties and other design features (but also by the character of materials, gravity, transportation, economics, etc.). For example, stylistic properties: Gothic, Classical, Baroque, Rococo, Neoclassical, Modernist, eclectic. It may be bold or timid, traditional or innovative, inspiring or depressing. I intend to use all of these terms to pick out aesthetic features, at least on some occasions of their use. And to an aesthetic experience or judgment of a facade as Gothic, for example, the actual date at which it was built, information about the design process and the people who participated in it, and its location are as relevant as 
the shape of the windows. Notice that, though we might refer to a contemporary building made of glass and steel as "Gothic," we might also refer to it as "pseudo-gothic" or "contemporary gothic" and so on, terms for aesthetic features that no fourteenth-century building could display. That is, a thing's various relations or relational properties - its histories and historically emergent properties - are potentially aesthetic features of it, depending on the purpose and context of interpretation.

Again, in my view, aesthetic features of an object - its shape, let us say, and the ways that shape means within a culture - are no more subjective than any other qualities of an object, for example its weight; indeed it is not hard to imagine cases where weight itself is an aesthetic feature of an object. People can be simply wrong about the aesthetic features of an object: aesthetic features become evident in interpretations of an object, but these interpretations are called forth and constrained massively by the character of the object, its origin, its material, as well as by real and recalcitrant features of the culture from which it emerges and the discourses in which it appears.

That is, attributions of beauty are true only under conditions which implicate "the natural," "the linguistic," and "the subjective" in every case. What makes truth possible in this sense is that these aspects are not insular, but strands of the skein all snarled up together. If we said beauty is in the eye of the beholder as opposed to being in the objective world, we would be entirely confused. The eye of the beholder is itself in the objective world. If you do not believe me, pluck out your left eye and look at it. There it is! This is true of your "mental images," true of every aspect of human consciousness. In fact, the characteristic derangements of human consciousness derive from losing or severing some of these strands: someone tried to cut off the psychotic's knot.

I am moving with apparent insouciance between aesthetic objects, experiences, and truths, and objects, experiences and truths quite in general. That is not mere confusion. That a couch weighs 150 pounds is a fact about the couch; to know what it means, you would have to know what a pound is, more or less, and understand a decimal notation. That a pound or kilogram is a standard measure of weight has to do with environmental/physical features such as gravity, as well as with the evolution of the human body and the histories of the cultures where the measures arose - the mechanisms or practices, for example, by which measurements are standardized and disseminated. If we had been a thousand seventeen times as strong as we are, we would have 
a completely different system. But that does not make the weight of things subjective or a mere cultural construction, and it does not make an assertion about the weight of something an assertion about our language. The aesthetic features of a situation are potentially as external to us and to our practices and as epistemically available as any other aspects of the world, though there are special problems of subtlety or ambiguity in the case of ascriptions of some aesthetic features: notoriously, "beauty." Well, there are special problems in a lot of areas: think about how complex a matter it might be in a vexed case to show that a particular economic system is capitalist or socialist, or a particular color blue or green, for example. Depending on the circumstances, it can be hard or impossible to weigh something; depending on the instruments, you might get imprecise or inaccurate results, and so on.

Now, I am not sure what to do with properties in general in ontology, but allowing them in and for them to function non-problematically for a moment, we could also try to treat them according to the skein or snarl idea. So for example, I suggested that under certain circumstances, weight is an aesthetic property. That is one of the reasons building in stone is interesting or important, or the fact that a structure is made of stone is an aesthetic aspect of it. The weight of the pyramids, or the sheer fact that relative to the human body they are immensely heavy, is at once a physical/external, a political, and an aesthetic feature of the pyramids (politically, we might say, the weight is an expression of the immense strength of the Pharaoh, or his immense ability to annex the strength of others; cf. the Pentagon). Well every physical object has weight (I think!), so it follows that every thing that exists has an aesthetic property, or has properties that in the right circumstances/juxtapositions/connections can be considered aesthetic. Weight is chosen almost at random here, and in this way of thinking about it, in connection to certain lash-ups, every object has a myriad of aesthetic properties. These properties are as objective and as non-objective as any other real properties: they are as objective and as non-objective as weight, for example. The aesthetic is lashed to the weight properties, color properties, size properties, shape properties, and with the ways all of these interact with each other and with an entire physical/social/individual world.

Now, you might think that, for example, aesthetic features like beauty have no actual physical effects, or have such effects only in relation to perceivers. Of course, when I see Lauren Bacall or her image on the screen and think she is beautiful, this does have physical effects: I reorient my body, stare, blood rushes here and there, neurons fire, etc. But 
that is because of my experience of the image; these things are happening in a conscious experiencer experiencing this image. But if weight is an aesthetic property, then subjectivity is not required for an object to display an aesthetic property. If you dropped a pyramid on me, it would smash me flat as a pancake, and not because I was interpreting it in a certain way. Yet it would not crush me if I did not exist as a body of a certain kind. If my body was of another kind, maybe I could catch the pyramid and toss it back, or brush it off like a fly: different "subjects" have different responses.

So, let us entertain this idea: aesthetic properties are exactly as objective as any other properties, which is to say that they are features of a situation implicating many levels of "being": again, a physical object in a physical context, a set of social practices, a set of personal experiences. Delete the personal experience and you have deleted the beauty, not because beauty is subjective, but because subjectivity is one strand in this knot; when this strand is disentangled it ceases to be the particular knot that it is.

Perhaps after all this I had better say something about environmental aesthetics. First of all, the approach I am suggesting means that environmental aesthetics ought to be considered epistemically legitimate: it is engaged in discerning the real qualities of real things. Indeed, the reality of things is at the heart of one's respect for them; to say they are real is to acknowledge them. That is, to exist is to count one way and another: ontologically, epistemically, morally, aesthetically. And then the question is, in any particular case, how and how much? So values are in play in ontology from the outset, and in this case, of course, we cannot fail to broach questions of aesthetic value in particular.

But of course the approach I'm suggesting also issues some cautions. We are not some outside force pouring artificial materials into a natural atmosphere, hence damaging it; we are creatures doing what we do with and as the materials we find around us. I am not sure we could even make sense of the idea of "damage" without having at least two agencies, or an agency in juxtaposition with an object. But if we are hurting nature, of course this is nature hurting itself. Our "destruction of nature" is its own self-destruction, though I do not think that "destruction" is ultimately going to make sense here. And then a question might be: how if at all would such an insight - if it is an insight - change our practice with regard to particular environments or environmental issues?

That might be the question that would most interest you. I cannot really try to deal with it in any full-fledged way here or perhaps any- 
where. But I do want to suggest that a complete acknowledgment of our complete inherence in the natural world would indeed change some of our practices. We have to start with an insistence on acceptance or affirmation - this world, exactly as it is with us in it, is the world. We have to try actually to experience our connections, in detail, an experience that has withdrawn under the pressure of a bad metaphysics or a wrong self-image - self-congratulatory or self-loathing - of the social, artificial, human, as a different order or position within the whole. And one thing I am saying is that withdrawing our grandiosity about ourselves as either masters or stewards of nature, as its users, destroyers, preservers, does not require us to stop deploying values or trying to act in accordance with them.

We had better think about what the aesthetic properties of this ecosystem, network, skein, web, fabric, really are. For one thing it is not a steady state or really even cyclical system, though it displays all kinds of epicycles or loops; it is a volatile system. It is explosive: more like a Caravaggio than a Raphael. This is true of global temperatures, of course, and we are just one of many factors that have cooled or heated the atmosphere of this earth. This is, I am afraid, a reality to which we are going to have to reconcile ourselves, but it is an aesthetic reality among its other dimensions. We can no more produce a steady ("sustainable") state than we can detect one already out there, or detect an equilibrium or balance that we are disturbing. There is in nature exactly as much equilibrium as there actually is, with us in it. We are not dealing with an invariant or cyclical nature but a tangle in time, tangled time. We are in a situation/ juxtaposition/collection of ourselves and everything that is not us. It/us is in the process of shifting: growing or shrinking, heating up or cooling down, bringing up the volume or turning it down.

We have to understand the human effect of nature, in other words, as nature's effect on itself: of course, what else could it be if you have any tendency toward naturalism? The idea that we are called upon to save or redeem the earth is exactly as committed to an ontology of supernatural beings (namely, the people who endorse the idea) as is the idea that we are the masters of the world and can legitimately, for example, use members of other species however we see fit.

Even within the wacky pluralism and mere metaphor of which I have availed myself so far, I want to say that I remain committed to some sort of basic materialism; we have got to try to hold on to the materiality of an aesthetic experience in a forest or in a museum: we need to 
hold on to particular bodies and objects: that is part of what it would mean to love the world or for the world to be beautiful. Materiality is what makes the world funky, what makes it external to ourselves, but also what makes ourselves part of it.

Thoreau writes: "What is it to be admitted to a museum, to see a myriad of particular things, compared with being shown some star's surface, some hard matter in its home! I stand in awe of my body, this matter to which I am bound has become so strange to me [...] Think of our life in nature, - daily to be shown matter, to come in contact with it, - rocks, trees, wind on our cheeks! the solid earth! the actual world! Contact! Contact! Who are we? where are we ?"3 I think matter is mysterious and more or less adorable. Or it is a bitch, really, but I would not do aesthetics without it, not when every artistic process is a transformation of materials by person and person by materials.

In other words, to begin with, art has to be conceived in terms of human/nonhuman assemblages: conceptual art is all very nice, but there really is no private art any more than there is a private language, and the work of art is a development in materials, an array of "facts": human/nonhuman material assemblages or skein regions; acts of communication among people and things. All art is environmental art in the sense that it all consists of processes within ecosystems, as well as reflecting objects, expressing emotions, and so on: art is above all a material intervention. This is rather a precious thing to say about a Brancusi sculpture, perhaps (but perhaps not), but it is not at all precious with regard to the design of a city or an approach to land use. Both of these, of course, are of necessity engagements in aesthetic value as well as other values; they are sites at which dimensions or arrays of values converge or are actualized materially. Even to make land-use policies or to design buildings in the complete absence of a desire for beauty or a rejection of it - as in the architecture of Marxist dictatorships - is to manifest aesthetic commitments and to have real aesthetic-material results: really to change the shape of things.

Well, then we need to think about the values we deploy in transforming the world, and here the approach that I am suggesting is compatible in many ways with the tradition of environmentalism. But even acts of imagination and government policies are material transformations. Many values are in play, or they all are, and they themselves have to be conceived as inherent in the world. For example: do we want to live in an entirely humanized or technological environment? Or maybe out in the woods with Thoreau? Well neither of these is more 
natural or more material or more socially articulated than the other. And among other things we ought to think about the dimensions of beauty that open up to experience in each such context. Then we may choose. But the transformations are not merely personal; they are always a participation in, an alteration of, the whole.

So I am giving a plea for the materialization of art, and hence for the continuity of art with reality, the understanding of human making as an upwelling within nature, a series of natural transformations, tantamount to erosion or vegetation. On the other hand, the aesthetic dimension of nature - perhaps opened up within nature by human making, perhaps not - is factual: something we detect, a series of juxtapositions within the reality in which we and our artifacts and our world, in our entirety and theirs and its, are entangled.

\section{Notes}

1. Ralph Waldo Emerson,"History," Essays: First Series, in Emerson: Essays and Lectures (New York: Library of America, 1983), 254.

2. See, e.g. Bruno Latour, Reassembling the Social: An Introduction to Actor-Network Theory (Oxford: Oxford University Press, 2007).

3. Henry David Thoreau, The Maine Woods, in Thoreau (New York: Library of America, 1985), 646. 